\title{
GluN2B-NMDA Receptors in Alzheimer's Disease: What Do they Got to Do with AD?
}

\section{Li L and Zhou Q*}

School of Chemical Biology and Biotechnology, Peking University Shenzhen Graduate School, Shenzhen, P.R China

Alzheimer's disease (AD) is one of the most devastating and debilitating diseases affecting the aging population. Dementia is the most widely known deficit in AD patients, typically starts with loss of recent memory (minutes to hours), and progresses to loss of long-held memories and unable to recognize their loved ones. People with $\mathrm{AD}$ eventually lose their ability to take care of themselves. As a consequence the cost of care is huge, and it also takes a tremendous emotional toll on the family of $\mathrm{AD}$ patients. Two hallmarks of $\mathrm{AD}$ are senile plaques and neurofibrillary tangles, which are composed of amyloid $\beta$ (A $\beta$ ) and tau, respectively. Despite extensive search for effective treatment over the past decades, available drugs have limited efficacy without affecting the course of AD. For example, cholinesterase inhibitors (such as Aricept) enhance the function of cholinergic functions by elevating the concentration of acetylcholine and improve memory functions moderately [1]. Memantine, a NMDA subtype glutamate receptor blocker, is used in treating moderate to severe AD patients, although its mechanism is still in debate [2]. Significant efforts have been devoted to curb the production of $A \beta$ or enhance its clearance, with the aim to reduce the plaque accumulation in the brain. Antibodies that bind $\mathrm{A} \beta$ to increase their removal have received a lot of attention. Although recent clinical trial results have been largely negative and disappointing [3], the recent exciting preliminary clinical trial results from Biogen has somewhat revived the hope in this approach.

NMDARs play critical roles in synaptic plasticity, memory functions and the refinement of neuronal connections during development [4]. It is well established that excessive activation of NMDARs can lead to neuronal death, generally defined as excitotoxicity. How NMDARs can mediate the above two apparently opposite functions has been a recent debate. There are two camps: one hypothesizes that NMDAR subunit composition determines whether NMDARs is beneficial or malicious (i.e., containing GluN2B leads to excitotoxicity while containing GluN2A is beneficial) [5], the other camp claims that it is their subcellular locations determines which action occurs (i.e., NMDARs at extrasynaptic regions mediate excitotoxicity while synaptic NMDARs are essential for physiological functions) [6]. It needs to be mentioned that extrasynaptic NMDARs (which are outside a synapse) can be activated only by glutamate spilled out of a synapse or by ambient glutamate present in the extracellular space. The majority of excitatory synapses on excitatory neurons in the neocortex and hippocampus are located on dendritic spines. Spine loss is highly correlated with the reduction in cognitive function in $\mathrm{AD}$ patients [7]. There is a large literature implicates that GluN2B-NMDARs play a critical role in neurodegeneration and in $A \beta$-induced synaptic dysfunction and synapse loss in $\mathrm{AD}$, while inhibiting GluN2B-NMDARs with selective antagonists appears to prevent or reverse some of the deficits $[4,8]$. Antagonists to GluN2B-NMDARs may have therapeutic values by providing neuroprotection and may improve cognitive function in $\mathrm{AD}$ patients. Besides $\mathrm{A} \beta$, Tau has been shown to be required for localizing fyn tyrosine kinase to dendritic spines, where it phosphorylates GluN2B-NMDARs and results in enhanced association between GluN2B-NMDAR with PSD-95 and subsequent downstream neurotoxic effects. Disrupting the interaction between GluN2B and PSD-95 in vivo improved memory functions and reduced premature death in $\mathrm{AD}$ mice [9]. However, the majority of evidence supporting $\mathrm{A} \beta$ 's role in degeneration in $\mathrm{AD}$ has been gathered from cultured neurons or acute brain slices in response to high concentrations of acutely applied $\mathrm{A} \beta$. Whether long-term in vivo treatment of $\mathrm{AD}$ mouse models with GluN2B antagonists is beneficial had not been reported: this is a key test in understanding the potential therapeutic value of GluN2B antagonists in AD. Therefore, there are two key questions need to be addressed to determine whether GluN2B-NMDARs have a critical contribution to AD: 1) Are GluN2B-NMADR functions altered in $\mathrm{AD}$ mice? 2) What are the long-term in vivo effects of selective GluN2BNMDARs in $\mathrm{AD}$ mice?

To address the above questions, we first examined their locations and contribution to synaptic function and plasticity in $\mathrm{AD}$ mice (PS2APP). Surprisingly, long-term potentiation (LTP) induced by theta burst stimulation was reduced in the presence of selective GluN2BNMDAR blocker Ro25-6981, suggesting that activation of GluN2BNMDARs contribute to synaptic plasticity [10]. In addition, these LTP-enabling GluN2B-NMDARs are present at perisynaptic regions. These perisynaptic locations are defined functionally in that NMDARs present here can be activated by high frequency burst synaptic inputs but not by low frequency single synaptic inputs. NMDARs present here can only be activated by glutamate that spills out of a synapse (such as during burst stimulation when glutamate uptake capacity is temporally overwhelmed). The density of extrasynaptic GluN2B-NMDARs is not altered in $\mathrm{AD}$ mice. Furthermore, there is no indication that the release profile of glutamate is altered in AD mice, as judged by the kinetics of recorded NMDAR currents; indicating that it is unlikely there is enhanced spillover of glutamate from a synapse [10]. These results painted a unexpected picture - that GluN2B-NMDARs likely play an important role to retain the brain's capacity to undergo synaptic plasticity than causing excitotoxicity. Whether long-term activation of these perisynaptic GluN2B-NMDARs could lead to excitotoxicity is unknown and difficult to address due to lack of proper tools to isolate and manipulate these receptors. In addition, there might be a possibility that these perisynaptic GluN2B-NMDARs may move to extrasynaptic locations to cause excitotoxicity as an animal ages. In [10], we only studied young AD mice, and hence there is a possibility that GluN2BNMDARs could be beneficial in young animals but became malicious and cause harm at older age.

To address whether long-term blocking GluN2B-NMDARs could

*Corresponding author: Dr. Qiang Zhou, School of Chemical Biology and Biotechnology, Peking University Shenzhen Graduate School, Shenzhen, P.R China, Tel: 8618503098108; E-mail: zhouqiang@pkusz.edu.cn

Received August 28, 2015; Accepted August 29, 2015; Published August 31, 2015

Citation: Zhou LL (2015) GluN2B-NMDA Receptors in Alzheimer's Disease: What Do they Got to Do with AD? J Neurol Disord 3: e118. doi:10.4172/23296895.1000e118

Copyright: ( 2015 Zhou LL. This is an open-access article distributed under the terms of the Creative Commons Attribution License, which permits unrestricted use, distribution, and reproduction in any medium, provided the original author and source are credited. 
be beneficial in $\mathrm{AD}$ mice, we used piperidine18 (Pip18), a potent and selective GluN2B-NMDAR antagonist [11]. Neither short-term (17 days) nor long-term (4 months) treatment with Pip18 in two AD mouse models (Tg2576 and PS2APP) resulted in any significant improvement in cognitive functions, as assayed using spatial learning (Morris water maze) and fear conditioning. In a model of an early onset $\mathrm{AD}$ (Ts65Dn, also a Down syndrome/mental retardation model), 2 week dosing of Ro25-6981 reduced performance in Barnes maze (a test on spatial memory) [11]. In addition, the typical spine loss associated with plaques in these $\mathrm{AD}$ mice was not affected [10]. In addition to episodic memory, we have also examined whether working memory could be improved with GluN2B antagonist. Injection of Ro25-6981 for 2 weeks did not affect working memory as measured using $\mathrm{Y}$ maze alteration [11]. It is possible that GluN2B antagonists need to be administered earlier (prior to formation of plaque) to curb pathogenesis. To address this, we treated 3 month-old AD mice with Pip 18 for 2 months, but did not observe any effect on spine loss associated with plaques [10]. As an indication of the bioavailability of Pip18 in the brain, both AD and wild type mice lost body weight, and increased anxiety-like behavior was observed in wild type mice. This lack of efficacy of GluN2B antagonists in AD models, both functionally and structurally, challenges the longheld expectation of the therapeutic potential for GluN2B-NMDAR antagonists in $\mathrm{AD}$.

The alterations of neural functions in wild type mice by GluN2B antagonists is worthy of further discussion. We found that acute Ro256981 treatment impaired Y-maze performance in wild type mice, and chronic treatment led to impaired in vitro gamma oscillations [11]. But we did not observe any benefit, either acutely or chronically, in Ts65Dn mice with these treatments. There are two other interesting and important findings in this study: 1) acute effects of GluN2B antagonists are often the opposite of chronic effects, both in vitro and in vivo; 2) activation of GluN2B-NMDARs on the GABAergic inhibitory interneurons increases the level of inhibition and hence contributes to the balance between excitation and inhibition in the neural circuitry. These results suggest that functions not altered in $\mathrm{AD}$ (and hence can be regarded as normal) may be affected by GluN2B antagonists, and these on-target undesirable side effect may cause potential liability for GluN2B antagonists.

Therefore, GluN2B-NMDARs do not appear to be a suitable target to treat $\mathrm{AD}$, then why are the above results/conclusions so different from those obtained using in vitro preparations and exogenous application of $\mathrm{A} \beta$ ? We suggest a few possibilities: 1$)$ the preparations used. It is very import to use appropriate models when studying disease mechanisms and identifying therapeutic targets. In this regard, acute application of high concentration of $A \beta$ onto developing neurons in vitro does not mimic the gradual increase in $A \beta$ concentrations in the mature/aged brain. 2) The notion of GluN2B-NMDARs causing excitotoxicity. Keep in mind that it is still a hypothesis that activation of GluN2B-NMDARs is a major cause of excitotoxicity in chronic neurodegenerative diseases (such as AD). Although this type of excitotoxicity may have significant contribution to neurodegeneration when there is an acute and large elevation in the extracellular glutamate concentration (such as during stroke), there is no direct evidence that similar process occurs in AD. As a matter of fact, our results actually point to the opposite. A recent study reported decreased GluN2B-NMDARs phosphorylation (Tyr1472) and reduced Src activity in young $\mathrm{AD}$ mice, suggesting reduced activity/ presence of GluN2B-NMDARs and likely reduced effect of GluN2B antagonists [12]. 3) GluN2B-NMDARs play critical roles in the proper functioning of neural circuitry. GluN2B-NMDARs are present on the inhibitory, GABAergic interneurons, and contribute to their physiological functions. Inhibiting GluN2B-NMDARs reduced synaptic inputs onto inhibitory neurons, altered the balance between excitation and inhibition, and resulted in altered neural network functions (such as gamma oscillations) [11]. Therefore, when interpreting the effects of GluN2B antagonists, one should not focus too much on the reduced excitotoxicity as if this is the only or main effect. Rather, we have to consider their effects on brain function and circuitry as a whole. Therefore, we need to change from an excitatory neuron-centric view to a more complete view of the circuitry when considering therapeutic values of GluN2B antagonists. In addition, when inhibition is altered (such as by GluN2B antagonists), the acute and long-term effects may not be the same since altered inhibition may drive reorganization of neural circuitry. Thus, the chronic effects of GluN2B antagonists cannot be readily deduced or extrapolated from their acute effects.

Why memantine is an approved AD drug? How memantine works in $\mathrm{AD}$ is still in debate. Various hypotheses have been proposed, from reducing excessive tonic activation but preserving phasic, physiological activation of NMDARs, to preferentially inhibiting GluN2C/2DNMDARs which are more abundantly present on inhibitory neurons [11]. When discussing the efficacy of AD treatment in animal models, improving cognitive function almost always take the center stage. Although improved cognitive function is the most desirable outcome, it can be achieved through many different venues. There are a range of other alterations associated with $\mathrm{AD}$, including neuropsychiatric issues (such as depression, anxiety, agitation, etc). Certain evidence suggests that memantine may reduce agitation and aggression [13]. Alleviating psychiatric problems can improve the quality of life in $\mathrm{AD}$ patients and likely their cognitive ability as well.

There has been a lot of work on GluN2B-NMDARs in the context of $\mathrm{AD}$, with the hope that they may be a viable $\mathrm{AD}$ target. This is an attractive idea especially considering there are a range of potent, selective GluN2B antagonists with excellent pharmacokinetic properties [1416]. Thus, it is surely disappointing that this may seem quite unlikely, at least from the point of improving cognitive function and saving synapse. Our recent results highlight the challenge of treating a complex disease with a protracted time course, such as AD. It has also shown us convincingly that a disease with system-wide changes cannot be comprehensively mimicked using cellular or synaptic models. Diseasemodifying therapeutic interventions need to affect many aspects of the nervous system.

\section{References}

1. Di Santo SG, Prinelli F, Adorni F, Caltagirone C, Musicco M (2013) A metaanalysis of the efficacy of donepezil, rivastigmine, galantamine, and memantine in relation to severity of Alzheimer's disease. J Alzheimers Dis 35: 349-361.

2. Kotermanski SE, Johnson JW (2009) Mg2+ imparts NMDA receptor subtype selectivity to the Alzheimer's drug memantine. J Neurosci 29: 2774-2779.

3. Panza F, Solfrizzi V, Imbimbo BP, Logroscino G (2014) Amyloid-directed monoclonal antibodies for the treatment of Alzheimer's disease: the point of no return? Expert Opin Biol Ther 30: 1-12.

4. Paoletti P, Bellone C and Zhou Q (2013) NMDA receptor subunit diversity: impact on receptor properties, synaptic plasticity and diseases. Nat Rev Neuroscience 14: 383-400.

5. Lai TW, Shyu WC, Wang YT (2011) Stroke intervention pathways: NMDA receptors and beyond. Trends Mol Med 17: 266-275.

6. Hardingham GE, Bading H (2010) Synaptic versus extrasynaptic NMDA receptor signaling: implications for neurodegenerative disorders. Nat Rev Neurosci 11: 682-696.

7. Terry RD, Masliah E, Salmon DP, Butters N, DeTeresa R, et al. (1991) Physical basis of cognitive alterations in Alzheimer's disease: synapse loss is the major correlate of cognitive impairment. Ann Neurol 30: 572-580. 
Citation: Zhou LL (2015) GluN2B-NMDA Receptors in Alzheimer's Disease: What Do they Got to Do with AD? J Neurol Disord 3: e118. doi:10.4172/23296895.1000 e118

8. Zhou Q, Sheng M (2013) NMDA receptors in nervous system diseases. Neuropharmacology 74: 69-75.

9. Ittner LM, Ke YD, Delerue F, Bi M, Gladbach A, et al. (2010) Dendritic function of tau mediates amyloid-beta toxicity in Alzheimer's disease mouse models. Cell 142: 387-397.

10. Hanson JE, Meilandt WJ, Gogineni A, Reynen P, Herrington J, et al. (2014) Chronic GluN2B antagonism disrupts behavior in wild-type mice without protecting against synapse loss or memory impairment in Alzheimer's disease mouse models. J Neurosci 34: 8277-8288.

11. Hanson J, Weber M, Meilandt W, Wu T, Luu T, et al. (2013) GluN2B antagonism affects interneurons and leads to immediate and persistent changes in synaptic plasticity, oscillations, and behavior. Neuropsychopharmacology 38: 12211233.

12. Mota SI, Ferreira IL, Valero J, Ferreiro E, Carvalho AL, Oliveira CR, Rego
AC (2014) Impaired Src signaling and postsynaptic actin polymerization in Alzheimer's disease micehippocampus - Linking NMDA receptors and the reelin pathway. Exp Neurol 261: 698-709.

13. Wilcock GK, Ballard CG, Cooper JA, Loft H (2008) Memantine for agitation/ aggression and psychosis in moderately severe to severe Alzheimer's disease: A pooled analysis of 3 studies. J Clin Psychiatry 69: 341-348.

14. Mony L, Kew JN, Gunthorpe MJ, Paoletti P (2009) Allosteric modulators of NR2B-containing NMDA receptors: molecular mechanisms and therapeutic potential. Br J Pharmacol 157: 1301-1317.

15. Riaza Bermudo-Soriano C, Perez-Rodriguez MM, Vaquero-Lorenzo C, BacaGarcia E (2012) New perspectives in glutamate and anxiety. Pharmaco Biochem Behav 100: 752-774.

16. Kaplan GB, Moore KA (2011) The use of cognitive enhancers in animal models of fear extinction. Pharmacol Biochem Behav 99: 217-228. 